\title{
Correlation of Site and Size of Tympanic Membrane Perforation and Middle Ear Air Space Volume with Magnitude of Hearing Loss
}

\author{
Ramandeep Singh Virk ${ }^{1}$ Krishan Kudawla ${ }^{1}$ Sandeep Bansal ${ }^{1} \quad$ Ramya Rathod $^{1}$ Samarendra Behera ${ }^{1}$ \\ ${ }^{1}$ Department of Otolaryngology, Head \& Neck Surgery, Post \\ Graduate Institute of Medical Education and Research (PGIMER), \\ Chandigarh, India \\ Address for correspondence Ramandeep Singh Virk, MS, \\ Department of Otolaryngology and Head \& Neck Surgery, Post \\ Graduate Institute of Medical Education and Research (PGIMER), \\ Sector-12, Chandigarh 160012, India (e-mail: virkdoc@hotmail.com).
}

Ann Otol Neurotol ISO 2019;2:10-15

\section{Abstract}

Keywords

- air-bone gap

- chronic otitis media

- various sites and sizes of perforation
Introduction The effects of tympanic membrane perforations on middle ear sound transmission are not well characterized, largely because ears with perforations typically have additional pathological changes. It has been established that the larger the perforation, the greater is the hearing loss $(\mathrm{HL})$.

Aim This study aimed to correlate the location and size of tympanic membrane perforation and middle ear air space volume with the magnitude of $\mathrm{HL}$ in patients with tubotympanic or inactive mucosal type of chronic otitis media (COM).

Materials and Methods A prospective clinical study of patients with tympanic membrane perforations due to COM and without any other ear disease and who attended the Otolaryngology services at our institute between July 2010 and December 2011 was conducted. A total of 300 ears were evaluated by performing otoendoscopy, followed by photo documentation and audiological investigations (pure-tone audiometry and tympanometry). Tympanic membrane perforations were categorized based on their size and location, and the mean air-bone $(A B)$ gap between the various types of perforations was compared and statistically analyzed with significance level of $p<0.05$. Results Out of 300 ears, maximum number of ears $(n=124,41.3 \%)$ had large-sized perforations $\left(>30 \mathrm{~mm}^{2}\right)$ that had a maximum mean $\mathrm{AB}$ gap of $26.43 \mathrm{~dB}$, and minimum number of ears $(n=60,20 \%)$ had small-sized perforations $\left(0-9 \mathrm{~mm}^{2}\right)$ that had minimum mean $A B$ gap of $9.12 \mathrm{~dB}$. The remaining were medium-sized perforations that had mean $A B$ gap of $16.13 \mathrm{~dB}$. Depending on the location, maximum were central perforations ( $n=198$, $66 \%$ ) and minimum were anterosuperior (AS) perforations ( $n=9,3 \%$ ). Based on the middle ear volume on tympanometry, maximum ears were of low-volume group ( $n=246$, $92 \%$ ) that had larger mean $A B$ gap of $19.96 \mathrm{~dB}$ HL when compared with the high-volume group ( $n=24,8 \%$ ) with $11.80 \mathrm{~dB} \mathrm{HL}$. AB gap was maximum at lower frequencies and decreased with increase in frequencies except at $4,000 \mathrm{~Hz}$, that is, $56.9 \mathrm{~dB} \mathrm{HL}$ at $250 \mathrm{~Hz}$, 42.6 at $500 \mathrm{~Hz}, 41.5$ at $1,000 \mathrm{~Hz}, 32.4$ at $2,000 \mathrm{~Hz}$, and 49.5 at $4,000 \mathrm{~Hz}$.

Conclusion $\mathrm{HL}$ increases as the area of tympanic membrane perforation increases. There is an inverse relationship between $\mathrm{HL}$ and middle ear air space volume. Comparing the small-sized perforations at different sites with the middle ear volume being low, it was found that posterosuperior (PS) perforations had 4 to $7 \mathrm{~dB}$ greater $\mathrm{HL}$ than $\mathrm{AS}$ and anteroinferior (Al). However, the relationship was statistically insignificant. The phase cancellation effect of round window causing greater $\mathrm{HL}$ in posteroinferior (PI) perforations does not exist in small- and medium-sized perforations. $\mathrm{HL}$ is greater at lower frequencies and less at higher frequencies. received

February 13, 2019

accepted

May 16, 2019

published online

July 16,2019
Copyright $\odot 2019$ Indian Society of Otology
License terms

() (1) $\circledast$ 


\section{Introduction}

Tympanic membrane (TM) serves several functions such as conduction of sound waves across the middle ear, protection of the middle ear cleft from infections, and shielding of the round window from direct sound waves, which is referred to as "round window baffle." 1 This shield is necessary to create a phase differential across the oval and round windows. The effect of the enhanced ratio of the TM surface area to that of the oval window increases the sound pressure by approximately $27 \mathrm{~dB}$, and the lever action of ossicles contributes to approximately $3 \mathrm{~dB} .2,3$

TM perforations can be classified based on their size, site, and etiology. TM perforation can result from many causes such as trauma, acute otitis media, and chronic otitis media (COM). ${ }^{4}$ Chronic suppurative otitis media has been an important cause of middle ear disease and is the most important cause of hearing loss (HL) in developing countries such as India. Poor and overcrowded living conditions, poor hygiene, and poor nutrition have been suggested as a basis for the widespread prevalence of chronic suppurative otitis media in developing countries. ${ }^{5}$ It is an inflammatory process of the mucoperiosteal lining of the middle ear space and the mastoid. ${ }^{6}$ COM is defined as chronic otorrhea through a nonintact (perforated) TM of greater than 6 weeks' duration unresponsive to oral and ototopical medications. ${ }^{5} \mathrm{COM}$ is also classified into active mucosal, inactive mucosal, active squamous, inactive squamous, and healed type. ${ }^{6}$

The effects of TM perforations on middle ear sound transmission are not well characterized largely because ears with perforations typically have additional pathological changes. ${ }^{5}$ It has been studied that the larger the perforation, the greater is HL. A total perforation of TM would lead to a loss in the transformer action of the middle ear. ${ }^{7}$ The site of the perforation is believed to have a significant effect on magnitude of HL. ${ }^{8}$ For instance, the posterior quadrant perforations are worse than the anterior ones because of the direct exposure of the round window to the sound waves, and perforations at or near the site of TM attachments to the manubrium have more severe effects than those of comparable size at different sites. ${ }^{9}$ However, some studies show that there is no significant effect associated with location of perforation. ${ }^{7,8,10} \mathrm{COM}$ can lead to conductive HLs of as much as $60 \mathrm{~dB}$, especially when the ossicles are involved, which constitutes a serious handicap. ${ }^{11}$ Perforations cause a loss that depends on frequency, perforation size, and middle ear air space volume. Perforation-induced losses are greatest at the lowest frequencies and generally decrease as frequency increases. ${ }^{5}$ Perforation size is an important determinant of the loss; larger perforations result in larger HLs. The volume of the middle ear air space (combined tympanic cavity and mastoid air volume) is also an important parameter that determines the amount of $\mathrm{HL}$ caused by a perforation. ${ }^{12}$

The idea that middle ear volume affects HL caused by a perforation has several clinical implications. It provides an explanation for the variability in HL that is observed clinically in patients with otherwise similar perforations. Also, it can account for fluctuations in the HL with the same perforation in a given patient during active disease when the middle ear volume might be expected to vary because of mucosal edema or accumulation of fluid within the middle ear spaces. This explains the clinical observation that TM perforations in patients with canal wall down mastoidectomy cavities have typically greater $A B$ gaps than similar-sized perforations in normal ears, because ears with canal wall down mastoidectomy cavities have smaller middle ear volumes.

Mehta et al speculated that the importance of location may have resulted from attempts to explain differences in $\mathrm{HL}$ with similar-sized perforations that were caused by inter-ear differences in middle ear volumes. ${ }^{13}$

The aim of this study was to correlate the location and size of TM perforation and middle ear air space volume with the magnitude of $\mathrm{HL}$ in patients with tubotympanic or inactive mucosal type of COM.

\section{Materials and Methods}

This cross-sectional study was conducted in the Department of Otolaryngology and Head \& Neck Surgery, Post Graduate Institute of Medical Education and Research, Chandigarh, India. All consecutive patients with COM (tubotympanic or inactive mucosal type) attending the Otolaryngology services from July 2010 to December 2011 were taken as study population. A total of 300 ears of patients with chronic otitis were studied. Informed and written consent of all the participating patients was taken, and the institute ethics committee approval was sought before commencing the study.

Inclusion criteria included patients of age between 15 and 45 years with tubotympanic or inactive mucosal type of COM with only mild to moderate conductive $\mathrm{HL}$, that is, up to 55 $\mathrm{dB}$ HL according to the World Health Organization (WHO) grading of hearing impairment, having good general physical condition, and not having any evidence of active infection in nose, throat, and paranasal sinuses.

Patients with active ear disease, attic retraction or perforation, presence of cholesteatoma or granulations, with sensorineural HL and history of trauma to the TM or prior attempts of its repair were excluded from the study.

Photo documentation with help of an otoendoscope was performed by recording a digital image of the perforation next to a scale, as shown in - Figs. 1 and $\mathbf{2}$. Then, the percentage area of the perforation was calculated using java image software. The following equation was generated: $\mathrm{P} / \mathrm{T} \times 100 \%$ = percentage perforation, where $P$ is the area (in pixels 2 ) of the TM perforation and $\mathrm{T}$ is the total area (in pixels 2 ) for the entire TM (including the perforation). ${ }^{14}$ Then area in square millimeter was calculated taking mean adult normal TM area as $85 \mathrm{~mm}^{2} .1,15$

The perforation was divided into three categories depending on the area of TM perforation: small $\left(0-8 \mathrm{~mm}^{2}\right)$, medium $\left(9-30 \mathrm{~mm}^{2}\right)$, and large $\left(>30 \mathrm{~mm}^{2}\right)$. Each patient underwent pure-tone threshold audiometry at frequencies 250, 500, $1,000,2,000$, and $4,000 \mathrm{~Hz}$. Air and bone conduction thresholds were determined. Contralateral ear was always masked while determining the bone conduction thresholds, whereas for air conduction thresholds, contralateral ear was masked if 


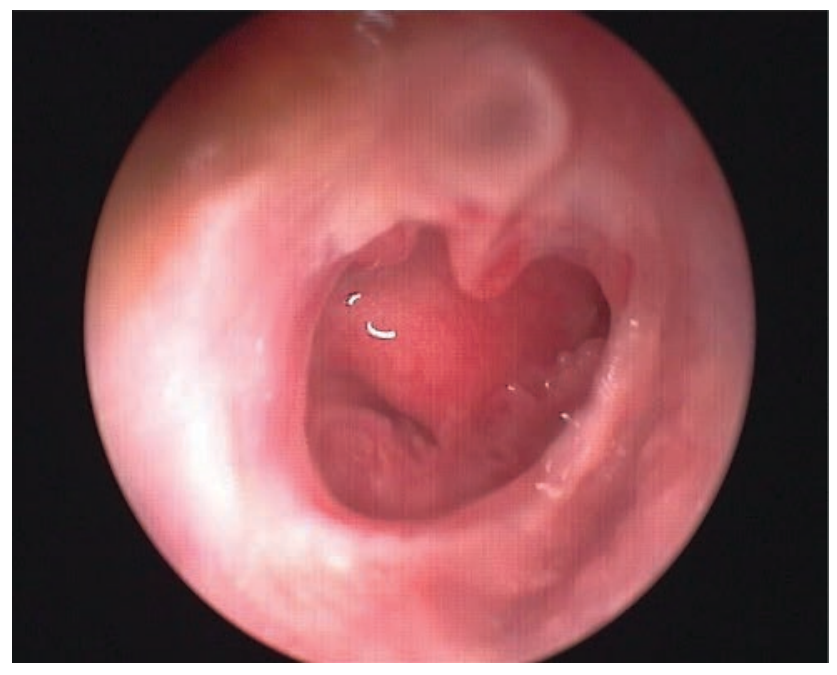

Fig. 1 Otomicroscopic picture of right tympanic membrane with central perforation of area $42 \mathrm{~mm}^{2}$ (large size), with air-bone gap of $18 \mathrm{~dB} \mathrm{HL}$ in low-volume group.

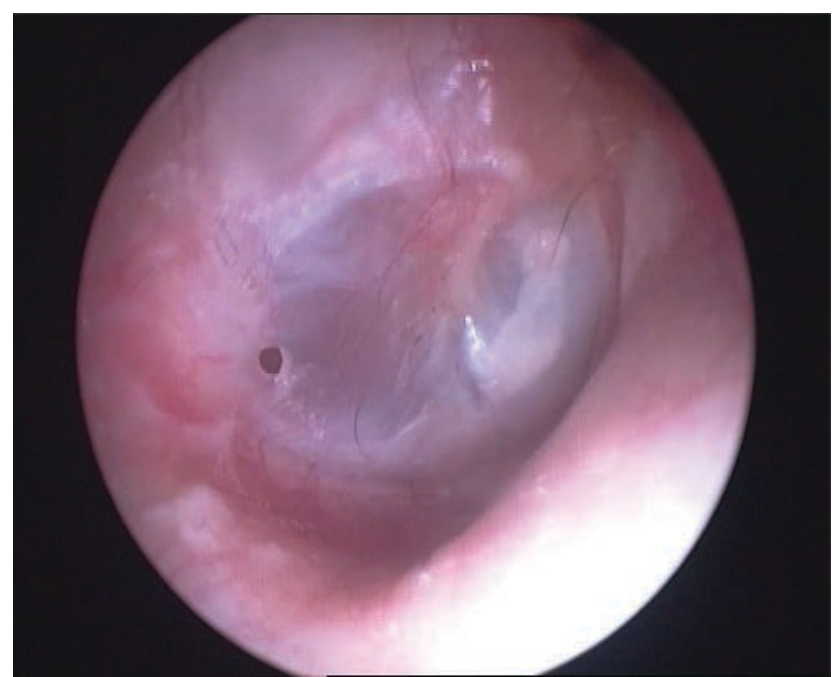

Fig. 2 Otomicroscopic picture of right tympanic membrane of the patient with posteroinferior of area $1 \mathrm{~mm}^{2}$ (small size) with no hearing loss.

the test ear was comparatively the poorer one and if sounds of $\geq 45 \mathrm{~dB}$ were used for the tests. The mean $\mathrm{HL}$ was calculated through the pure-tone average taken at 500,1,000, and $2,000 \mathrm{~Hz}$ for each site of perforation. ${ }^{1-5,13}$

Thereafter, patients underwent tympanometry to measure the volume of middle ear and mastoid space of ipsilateral ear, which is the combined volume of external auditory canal, middle ear, and mastoid air space. Then patients underwent tympanometry of contralateral ear (with intact TM), which is the volume of the external auditory canal. Subtracting the volume of ipsilateral ear with that of contralateral ear provided the volume of middle ear and mastoid space of the ipsilateral ear. For patients with bilateral perforations, the mean of ear canal volume of unilateral perforation was used. The ears were divided into two subgroups: small $(<4.3 \mathrm{~mL})$ and large $(>4.3 \mathrm{~mL}){ }^{13}$
The comparison was made between

1. Mean air-bone (AB) gap between small- and large-sized perforations, and small- and medium-sized perforations at different frequencies.

2. Mean AB gap of small- and large-volume groups.

3. Mean AB gap of perforation of different locations (anterosuperior [AS], posterosuperior [PS], anteroinferior [AI], posteroinferior [PI], and central) but of similar size and middle ear volume within this study population.

\section{Results}

A total of 246 patients (males 131, females 115) ( - Tables 1, 2) were included in the study with 54 bilateral $(n=54)$ and 192 unilateral ( $n=192$ ) cases, with total number of cases (ears) being 300. Maximum cases ( $n=124,41.6 \%$ ) were of large-sized perforations ( $>30 \mathrm{~mm}^{2}$ ), and minimum cases $(n=60,20 \%)$ were of small-sized perforations group (0-9 $\left.\mathrm{mm}^{2}\right)$, as shown in -Fig. 3. Maximum cases were of central perforations ( $n=198,66 \%$ ), and minimum cases were of AS perforations ( $n=9,3 \%$ ). Large-sized perforations were found to have maximum mean $\mathrm{AB}$ gap of $26.43 \mathrm{~dB}$, and small-sized perforations were found to have minimum mean $A B$ gap of $9.12 \mathrm{~dB}$. Medium-sized perforations had mean $A B$ gap of $16.13 \mathrm{~dB}$. Maximum cases were of low-volume group ( $n=246,92 \%$ ). High-volume group had $8 \%$ cases. In the remaining 34 cases, volume could not be calculated as seal could not be achieved during tympanometry, so they were excluded from the study of correlation of air space volume with HL.

The correlation of mean $A B$ gap with perforation size is shown in -Fig. 4. The low-volume group had larger mean AB gap (19.96 dB HL) than the high-volume group (11.80 dB $\mathrm{HL})$. The $t$-test was applied, and the difference was found to be statistically significant with a $p$-value $=0.004$. The mean $\mathrm{AB}$ gap was highest in central perforation (23.14 dB HL) and lowest in AS perforation (7.5 dB HL). On applying ANOVA (analysis of variance) test, this difference was found to be statistically significant with $p=0.00$.

\section{Correlation of Air-Bone Gap with Site of Perforation in Low Volume, Medium Area of Perforation}

In low-volume and medium-area category, the maximum mean $A B$ gap was in central perforation (19.6 dB) and minimum $A B$ gap was in PS perforations, that is, $11 \mathrm{~dB}$, where

Table 1 Sex distribution of cases in the study

\begin{tabular}{|l|l|l|l|}
\hline & Male & Female & Total \\
\hline Number & 131 & 115 & 246 \\
\hline Percentage & 53.2 & 46.7 & \\
\hline
\end{tabular}

Table 2 Age distribution of the study group

\begin{tabular}{|l|l|l|l|}
\hline Age groups (y) & $\mathbf{1 5 - 2 5}$ & $\mathbf{2 5 - 3 5}$ & $\mathbf{3 5 - 4 5}$ \\
\hline Number & 116 & 64 & 66 \\
\hline Percentage & 47 & 26 & 26.8 \\
\hline
\end{tabular}




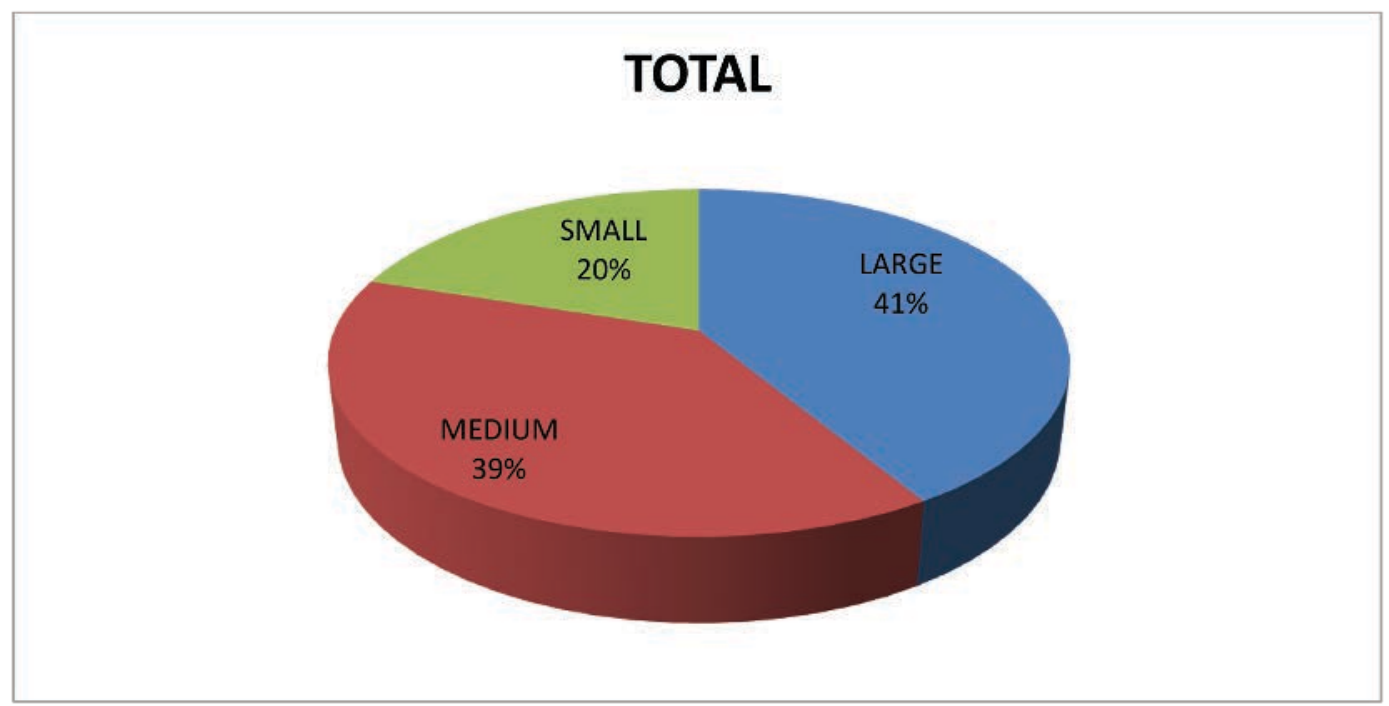

Fig. 3 Pie diagram showing distribution of cases into large size (> $\left.30 \mathrm{~mm}^{2}\right)$, medium size $\left(9-30 \mathrm{~mm}^{2}\right)$, and small size $\left.(0-9 \mathrm{~mm})^{2}\right)$.

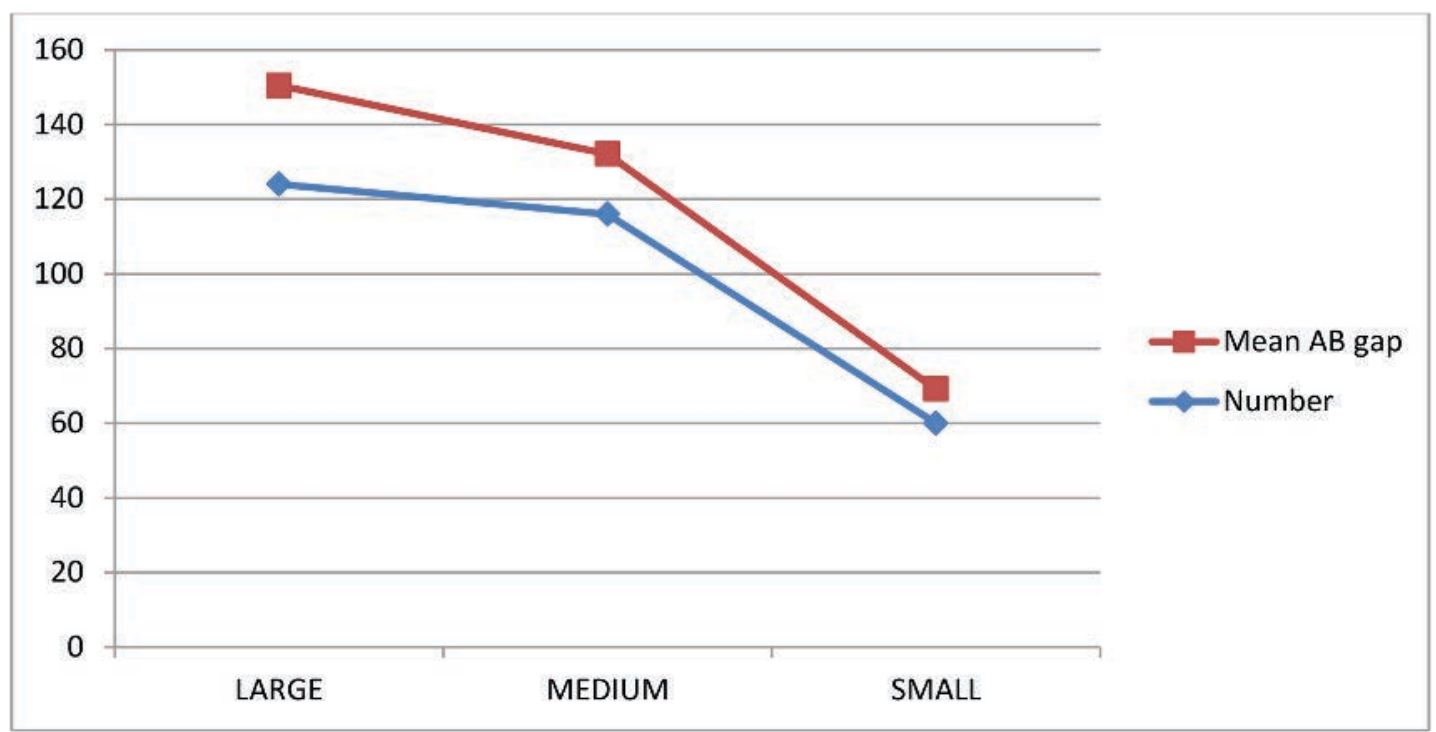

Fig. 4 Line diagram showing correlation of mean air-bone (AB) gap with perforation size.

it was $13 \mathrm{~dB}$ in $\mathrm{AI}, \mathrm{AS}$, and PI segments. ANOVA test was applied, which showed that the differences were statistically significant with $p=0.005$.

\section{Correlation of Air-Bone Gap with Site of Perforation in Low Volume, Small Area of Perforation}

In low-volume, small perforation area, the mean $\mathrm{AB}$ gap was highest in PS perforation (15 dB HL) and lowest in PI perforations (0.5 dB HL). It was $9.5 \mathrm{~dB} \mathrm{HL}$ in central perforation cases, 8 in AS, and 13 in AI cases. On applying ANOVA test, the difference was found to be statistically significant with $p=0.001$.

\section{Correlation of Air-Bone Gap with Site of Perforation in Low Volume, Large Area Group}

In low-volume, large-area category, 106 cases were of central perforations and only 1 case was of PI perforation. No cases of other site of perforation were seen in this group. Mean AB gap was $26 \mathrm{~dB}$ in central perforation cases and 18 in one PI case. On applying ANOVA test, it was found that the correlation was statistically nonsignificant with $p=0.499$. The groups were incomplete in high-volume low-area, high-volume medium-area, and high-volume large-area subgroups. Therefore, comparison could not be done in these subgroups.

\section{Frequency Distribution of Air-Bone Gaps}

$\mathrm{AB}$ gaps were maximum at lower frequencies and decreased with increase in frequencies except at $4,000 \mathrm{~Hz}$. It was 56.9 dB HL at $250 \mathrm{~Hz}, 42.6$ at $500 \mathrm{~Hz}, 41.5$ at $1,000 \mathrm{~Hz}, 32.4$ at $2,000 \mathrm{~Hz}$, and 49.5 at $4,000 \mathrm{~Hz}$.

\section{Discussion}

The primary mechanism of HL by a TM perforation is a reduction in middle ear sound transmission caused by a loss in sound pressure difference across the TM. On analyzing 
various aspects of perforation, we found that the HL depends on the size, site, and middle ear air space volume.

\section{Effect of Size of Perforation}

All ears with perforation in our study were divided into three groups: (I) small-sized perforation $\left(0-9 \mathrm{~mm}^{2}\right)$, (II) medium-sized perforation (9-30 $\left.\mathrm{mm}^{2}\right)$, and (III) large-sized perforation $\left(30 \mathrm{~mm}^{2}\right)$. Maximum number of ears were found in group III $(n=124,41.6 \%)$, followed by group II $(n=116$, $30 \%)$. Minimum number of ears were in group $\mathrm{I}(n=60,20 \%)$. On the contrary, Mehta et $\mathrm{al}^{13}$ reported maximum cases 30 (48.35\%) in small-area group and minimum cases 7 (11.2\%) in large-area group, out of the total of 62 ears, which is not in agreement with our study. As per a study by Pannu et al, ${ }^{16}$ $47 \%$ patients were categorized to group I, 34\% to group II, and remaining $19 \%$ to group III. The mean $A B$ gap in small-, medium-, and large-sized perforations were 9,16 , and $26 \mathrm{~dB}$, respectively. The difference was found to be statistically significant with $p=0.00$. As the size of perforation increased, the $\mathrm{AB}$ gap also increased.

Similarly, Ibekwe et $\mathrm{al}^{3}$ also found a positive correlation of perforation size with the magnitude of $\operatorname{HL}(p=0.01)$. Similarly, Mehta et $\mathrm{al}^{13}$ reported that the difference in $\mathrm{AB}$ gaps in small size $\left(0-9 \mathrm{~mm}^{2}\right)$ and large size $\left(>30 \mathrm{~mm}^{2}\right)$ and small size and medium size $\left(9-30 \mathrm{~mm}^{2}\right)$ were significant $(p<0.05)$ at $1,000,2,000$, and $4,000 \mathrm{~Hz}$. They also concluded that $A B$ gap increased with increase in the size of TM perforations. Pannu et $a{ }^{16}$ also found that mean $A B$ gap increased statistically significantly as the perforation size increased. Nepal et $\mathrm{al}^{17}$ also found that the hearing is directly proportional to the perforation size. Matsuda et $\mathrm{al}^{18}$ in their study also found that the larger the area of a traumatic perforation, the greater the conduction disturbance.

Mills et al ${ }^{19}$ also found a significant relationship between HL and perforation size, with a strong trend for HL to increase as the perforation size increased $(p=0.01)$. This correlation is due to decrease in sound pressure difference across the TM with the increase in size of the TM perforation.

\section{Effect of Middle Ear Air Space Volume}

In our study, low-volume group had $92 \%$ cases and high-volume group had $8 \%$ cases. It was found that HL decreased as the volume of middle ear air space increased with $p=$ 0.004. Low-volume group had mean $A B$ gap of $19.9 \mathrm{~dB}$, and high-volume group had mean $\mathrm{AB}$ gap of $11.8 \mathrm{~dB}$, which was found to be statistically significant with $p=0.004$. Mehta et $\mathrm{al}^{13}$ also found that the mean $\mathrm{AB}$ gaps in the small-volume group were larger by 10 to $20 \mathrm{~dB}$ compared with those in the large-volume group. These differences were statistically significant ( $p<0.05$, Student's $t$-test) at $250,500,1,000$, and $4,000 \mathrm{~Hz}$.

Similarly, Voss et $\mathrm{al}^{10}$ found that perforations in two different ears that appeared identical could have losses that differed by up to $30 \mathrm{~dB}$, if the middle ear air space volumes differed substantially. The reason why middle ear volume should affect HL caused by a perforation is that for a given perforation, the resulting sound pressure within the middle ear space will vary inversely with the middle ear volume.

\section{Effect of Site of Perforation}

In our study, 32 (10.6\%) perforations were in AI segment, 9 (3\%) were in AS segment, 18 (6\%) in PS segment, and 41 (13.6\%) in PI segment. Two hundred (66.6\%) involved the central compartment. Maharajan et $\mathrm{al}^{5}$ found that large central perforation was seen in $60.50 \%$ of cases, close to the result of our study. Perforation in PS quadrant was the least common finding in their study, whereas in our study, the least common site of perforation was AS quadrant. Pannu et al ${ }^{16}$ reported 45 (38\%) perforations in the anterior quadrant, $35(29 \%)$ in the posterior quadrant, and $40(33 \%)$ in the multiple quadrant. Similarly, Mehta et $\mathrm{al}^{13}$ found that $27 \%$ perforations were anterior, $30 \%$ were posterior, and $43 \%$ affected both halves of the TM. Therefore, it can be concluded that central perforations are the most common site of TM perforations.

On analyzing the effect of location of perforation on hearing, it was found that the mean $A B$ gap was maximum in central perforations $(23 \mathrm{~dB})$ and minimum in PI perforations (9.2 dB). The difference in mean AB gap in central perforations was statistically significant with all other sites. However, it was also found that $60 \%$ of central perforations were of large size. In low-volume, medium-area group, a total of 91 cases were observed. The mean $\mathrm{AB}$ gap of $\mathrm{AI}, \mathrm{AS}$, and PI were found to be similar, that is, $13 \mathrm{~dB}$, and PS group had mean $\mathrm{AB}$ gap of $11 \mathrm{~dB}$. The values were found to be statistically significant $(p=0.005)$. This contradicts a widely held clinical view that perforations over the round window, that is, PI perforations, result in a greater HL due to the sound pressure acting at the round window, which diminishes the cochlear responses by the "phase cancellation effect." The reason for this observation is that the wavelengths of sounds at $<4 \mathrm{kHz}$ are larger than the middle ear dimensions, so the phase cancellation effect should theoretically be the same for all sites of perforations. This is in agreement with the experimental works of Voss et $\mathrm{a}^{10}$ and Mehta et $a \mathrm{al},{ }^{13}$ who found no effect of location of perforations on the pressure difference at oval and round window and their clinical studies, also showed that there was no difference in $\mathrm{HL}$ in anterior and posterior perforations having the same middle ear volume. These observations are contrary to the results of Pannu et al, ${ }^{16}$ Bhusal and Shrivastav, ${ }^{20}$ Durko and Latkowski, ${ }^{15}$ Nepal et al, ${ }^{17}$ and Yung. ${ }^{21}$

However, in our study, the medium-sized central perforations were found to have 7 to $9 \mathrm{~dB}$ greater mean $\mathrm{HL}$ than anterior or posterior perforations. This can be explained by the fact that medium-sized central perforation involves the handle of malleus. This concept was initially proposed by Ahmad and Ramani ${ }^{8}$ who observed that malleolar perforations had greater HL than that of similar-sized nonmalleolar perforations. In low-volume, small-area group, a total of 48 cases were studied. PI site was found to have less mean $A B$ gap than AI and PS sites by 12 to $14 \mathrm{~dB}$, which was found to be statistically significant $(p=0.001$ ). Similar to the observations in low-volume, medium-area group, this also showed that there was no round window phase cancellation effect on HL at different locations in small-sized perforations. It can thus be concluded that the round window phase cancellation effect causing greater HL in PI perforations does not exist. It was also found that PS group had maximum mean $A B$ gap of 
$15 \mathrm{~dB}$, which was 4 to $7 \mathrm{~dB}$ more than those in the AS and AI groups. However, the difference was found to be statistically nonsignificant $(p>0.05)$.

The most probable explanation is that the PS perforations are more prone, although not exclusively to the diseases such as ossicular discontinuity and cholesteatoma. ${ }^{22}$ This is in agreement with earlier observations by Ahmad and Ramani. ${ }^{8}$ It also confirms the findings by Ibekwe et $\mathrm{al}^{3}$ who found a statistically significant correlation in HL at PS perforations in chronic cases. The third subgroup that was studied was the low-volume large-area group. In this subgroup, there were 106 cases of central perforations and only 1 case of PI perforation with mean $\mathrm{AB}$ gap of 26 and $18 \mathrm{~dB}$, respectively. Therefore, correlations could not be done in this subgroup. The $p$-values were found to be nonsignificant.

Limitations of our study were that we did not evaluate the status of ossicles and the Eustachian tube functioning as we excluded the patients with HL more than the moderate conductive $\mathrm{HL}$, which is suggestive of ossicular chain involvement by the disease.

\section{Conclusion}

Hearing loss increases as the area of TM perforation increases, and it is greater at lower frequencies and less at higher frequencies. However, in small- and medium-sized perforations, the phase cancellation effect of round window causing HL in PI perforations does not exist. There is an inverse relationship between $\mathrm{HL}$ and middle ear air space volume. On comparing small-sized perforations with low middle ear volume at various sites, PS perforations were found to have greater hearing impairment than the others, which was statistically insignificant in our study and would require further studies with a greater number of ears with PS perforations to establish its significance on HL.

\section{Note}

This study was cleared by institution ethics committee.

\section{Funding \\ None.}

\section{Conflict of Interest}

None declared.

\section{References}

1 Ogisi FO, Adobamen P. Type 1 tympanoplasty in Benin: a 10- year review. Niger. Postgrad Med J 2004;11(2):84-87

2 Voss SE, Rosowski JJ, Merchant SN, Peake WT. Non-ossicular signal transmission in human middle ears: experimental assessment of the "acoustic route" with perforated tympanic membranes. J Acoust Soc Am 2007;122(4):2135-2153
3 Ibekwe TS, Nwaorgu OG, Ijaduola TG. Correlating the site of tympanic membrane perforation with hearing loss. BMC Ear Nose Throat Disord 2009

4 Chole R, Sudhoff HH. Chronic otitis media. In: Cummings Otolaryngology-Head and Neck Surgery. 5th ed. Philadelphia, PA: Mosby;2005:4985-4986

5 Maharjan M, Kafle P, Bista M, Shrestha S, Toran KC. Observation of hearing loss in patients with chronic suppurative otitis media tubotympanic type. Kathmandu Univ Med J (KUMJ) 2009;7(28):397-401

6 Bluestone CD. Epidemiology and pathogenesis of chronic suppurative otitis media: implications for prevention and treatment. Int J Pediatr Otorhinolaryngol 2018;42(3):207-223

7 American Academy of Otolaryngology Head and Neck. Perforated Ear Drum. [Internet]2005[cited March 2010]. Available athttp://www.entnet.org/HealthInformation/ perforatedEardrum.cfm

8 Ahmad SW, Ramani GV. Hearing loss in perforations of the tympanic membrane. J Laryngol Otol 1979;93(11):1091-1098

9 Ronald NJ, McRac RD, McCombe AW, Chronic suppurative otitis media. In: Key Topics in Otolaryngology and Head and Neck Surgery. 2nd ed. Wales: Bios Scientific Publishers2001;38-41

10 Voss SE, Rosowski JJ, Merchant SN, Peake WT. How do tympanic-membrane perforations affect human middle-ear sound transmission? Acta Otolaryngol 2001;121(2):169-173

11 Kruger B, Tonndorf J. Middle ear transmission in cats with experimentally induced tympanic membrane perforations. J Acoust Soc Am 1977;61(1):126-132

12 Merchant SN, Rosowski JJ, Auditory physiology and middle-ear mechanics. In: Glasscock ME, Guliya AJ, eds. Surgery of the Ear. 5th ed. Ontario: BC Decker; 2003:59-82

13 Mehta RP, Rosowski JJ, Voss SE, O’Neil E, Merchant SN. Determinants of hearing loss in perforations of the tympanic membrane. Otol Neurotol 2006;27(2):136-143

14 Ibekwe TS, Adeosun AA, Nwaorgu OG. Quantitative analysis of tympanic membrane perforation: a simple and reliable method. J Laryngol Otol 2009

15 Durko T, Latkowski B. Extrameatal myringoplasty in the treatment of tympanic membrane perforations [article in Polish] Otolaryngol Pol 1997;51(Suppl 25):274-277

16 Pannu KK, Chadha S, Kumar D. Preeti. Evaluation of hearing loss in tympanic membrane perforation. Indian J. Otolaryngol Head Neck Surg 2011;63(3):208-213

17 Nepal A, Bhandary S, Mishra SC, Singh I, Kumar P. The morphology of central tympanic membrane perforations. Nepal Med Coll J 2007;9(4):239-244

18 Matsuda Y, Kurita T, Ueda Y, Ito S, Nakashima T. Effect of tympanic membrane perforation on middle-ear sound transmission. J Laryngol Otol Suppl 2009;123(31, Suppl S31):81-89

19 Oluwole M, Mills RP. Tympanic membrane perforations in children. Int J Pediatr Otorhinolaryngol 1996;36(2):117-123

20 Bhusal CL, Shrivastav R. Correlation of hearing impairment with site of tympanic membrane perforation. J Inst Med 2005;27(2):91-96

21 Yung MW. Myringoplasty: hearing gain in relation to perforation site. J Laryngol Otol 1983;97(1):11-17

22 Oktay MF, Cureoglu S, Schachern PA, Paparella MM, Kariya $\mathrm{S}$, Fukushima $\mathrm{H}$. Tympanic membrane changes in central tympanic membrane perforations. Am J Otolaryngol 2005;26(6):393-397 